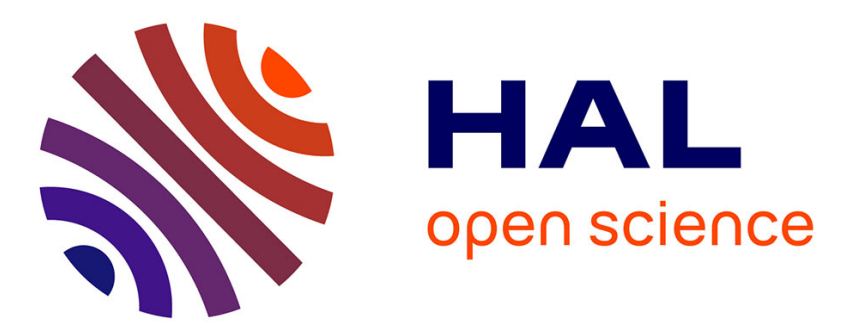

\title{
Impact of pump OSNR on noise figure for fiber-optical parametric amplifiers
}

\author{
A. Durécu-Legrand, C. Simonneau, D. Bayart, Arnaud Mussot, T. Sylvestre, \\ E. Lantz, H. Maillotte
}

\section{- To cite this version:}

A. Durécu-Legrand, C. Simonneau, D. Bayart, Arnaud Mussot, T. Sylvestre, et al.. Impact of pump OSNR on noise figure for fiber-optical parametric amplifiers. IEEE Photonics Technology Letters, 2005, 17, n 6, pp.1178-1180. hal-00097403

\section{HAL Id: hal-00097403 \\ https://hal.science/hal-00097403}

Submitted on 26 Aug 2013

HAL is a multi-disciplinary open access archive for the deposit and dissemination of scientific research documents, whether they are published or not. The documents may come from teaching and research institutions in France or abroad, or from public or private research centers.
L'archive ouverte pluridisciplinaire HAL, est destinée au dépôt et à la diffusion de documents scientifiques de niveau recherche, publiés ou non, émanant des établissements d'enseignement et de recherche français ou étrangers, des laboratoires publics ou privés. 


\title{
Impact of Pump OSNR on Noise Figure for Fiber-Optical Parametric Amplifiers
}

\author{
A. Durécu-Legrand, C. Simonneau, D. Bayart, Member, IEEE, A. Mussot, T. Sylvestre, E. Lantz, and H. Maillotte
}

\begin{abstract}
Electrical measurements of the noise figure of a fiberoptical parametric amplifier are presented and compared with optical measurements. The transfer of pump noise by four-wave mixing was clearly demonstrated. A numerical model was developed to simulate the transfer of pump noise and validated by these measurements. Using this model, we determine, for practical systems, a minimum required pump optical signal-to-noise ratio of $65 \mathrm{~dB}$.
\end{abstract}

Index Terms-Noise figure (NF), optical amplifiers, optical fiber communication, optical Kerr effect, parametric amplifiers.

\section{INTRODUCTION}

W ITH THE recent advances, it is now well-known that the fiber-optical parametric amplifiers (FOPAs) succeed in providing broad-band and high gain amplification. Very few studies are presently focusing on the noise performance. First, Blows and French showed that, in the case of a parametric pump constituted by a continuous source amplified by an erbium-doped fiber amplifier (EDFA), the output noise level of the FOPA is considerably reduced when the pump is filtered so as to remove any broad-band amplified spontaneous emission (ASE) noise present in the amplification band [1]. More recently, it was proved that rather than an optical measurement, an electrical measurement of the noise figure (NF) of the FOPAs was required to take into account other sources of noise than ASE [2]. Kylemark et al. numerically found that the NF is degraded by the remaining ASE under the pump peak [3]. In this letter, we present an electrical measurement method of the NF. With this method, we experimentally demonstrate the impact of the pump optical signal-to-noise ratio (OSNR) on the FOPA NF. Finally, we show that a pump OSNR as high as $65 \mathrm{~dB}$ is required to keep the NF to the quantum limit with a channel input power of $-21 \mathrm{dBm}$.

\section{MeAsurement Method of The Noise Figure}

The NF of an optical amplifier can be evaluated either optically from the measurements of the input and output OSNRs, or electrically. However, optically, only broad-band noise, like ASE, is detected because of the resolution of optical spectrum

Manuscript received October 21, 2004; revised January 20, 2005.

A. Durécu-Legrand, C. Simonneau, and D. Bayart are with the Research and Innovation Department, Alcatel, Marcoussis F-91460, France (e-mail: anne.durecu@alcatel.fr).

A. Mussot, T. Sylvestre, E. Lantz, and H. Maillotte are with the Département d'Optique P.M. Duffieux, Institut FEMTO-ST,U.M.R CNRS/Université de Franche, Besançon Cedex 25030, France.

Digital Object Identifier 10.1109/LPT.2005.846559 analyzers (OSAs). An electrical measurement gives a more accurate estimation of the NF. The electrical NF is determined with a relative-intensity noise (RIN) analyzer and by the RIN subtraction technique [4]

$$
\mathrm{NF}_{\text {elec }}=\frac{P_{\text {in }}}{2 \cdot h \cdot \nu}\left(\mathrm{RIN}_{\text {out }}-\mathrm{RIN}_{\text {in }}\right)
$$

where $P_{\text {in }}$ is the input signal power, RIN $_{\text {in }}$ and RIN $_{\text {out }}$ are, respectively, the RIN measured at the amplifier input and output. The measured value of RIN is due to different types of noise: The actual RIN of the signal source but also the beating noise between the signal and any other optical noise

$$
\begin{aligned}
& \mathrm{RIN}_{\text {in }}= \frac{1}{P_{\text {in }}^{2}}\left(\frac{4 \cdot N_{\mathrm{in} / /} \cdot P_{\mathrm{in}}}{\Delta \nu}\right)+\mathrm{RIN}_{\text {source }} \\
& \mathrm{RIN}_{\text {out }}=\frac{1}{P_{\text {out }}^{2}}\left(\frac{4 \cdot G \cdot N_{\mathrm{in} / /} \cdot P_{\text {out }}}{\Delta \nu}\right. \\
&\left.\quad+\frac{4 \cdot N_{\mathrm{ampli} / /} \cdot P_{\text {out }}}{\Delta \nu}\right)+\mathrm{RIN}_{\text {source }}
\end{aligned}
$$

In these expressions, $G$ is the amplifier gain, $N_{\text {in// }}$ an input noise, and $N_{\text {ampli// noise generated in the amplifier responsible }}$ of the NF. The notation // means that only the polarization component of noise, parallel to the signal one, beats the signal.

In order to validate the electrical method, an experiment was set up to perform simultaneously optical and electrical measurements of noise in the well-known case of an EDFA. Output noise is only constituted by broad-band ASE which can be easily detected either optically or electrically. The output of the amplifier is divided by a coupler. On one port, an OSA measured the gain and the OSNR. On the other port, an optical filter with a 3-dB bandwidth of $0.2 \mathrm{~nm}$ selects the signal wavelength and a RIN analyzer measures the output RIN at a particular frequency. The RIN analyzer is calibrated and subtracts the thermal noise and the shot noise from the total noise. To determine the NF, levels of noise are first measured at the input of the amplifier then at the output. A fair agreement was found between both electrical and optical NFs; the discrepancy between both is about $0.35 \mathrm{~dB}$. These measurement techniques can be applied on a parametric amplifier provided we take into account the particularity of the one-pump FOPA, which is to produce polarized ASE contrary to EDFAs as pointed out in [6]. This assertion was experimentally checked out with the following experiment. At the output of the FOPA, a filter selected $0.2 \mathrm{~nm}$ in the signal band at the maximum of gain. No signal was injected in the FOPA and the 


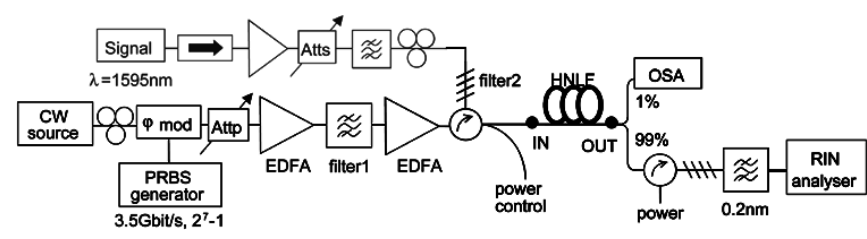

Fig. 1. Experimental setup for the measurement of the NF of the FOPA.

pump was filtered so that only ASE was detected. A polarization controller placed in front of a polarizer enabled to measure a polarization extinction ratio of $18 \mathrm{~dB}$ with an OSA. It corresponds to a degree of polarization of $98 \%$ so we can state that the ASE of the FOPA is fully polarized.

At high gain, the standard definition of NF is NF $=2 \cdot \mathrm{nsp}$. $(G-1) / G$, where nsp is the population inversion factor. For FOPA, the ASE is polarized so Pase $=n s p \cdot(G-1) \cdot h \nu \cdot d \nu$ and the optical NF is related to the total ASE power $P_{\mathrm{ASE}}$ as follows:

$$
\mathrm{NF}_{\mathrm{FOPA}}=\frac{2 P_{\mathrm{ASE}}}{h \nu \Delta \nu G}
$$

whereas, in the case of an EDFA, the ASE is not polarized so Pase $=2 \cdot \operatorname{nsp} \cdot(G-1) \cdot h \nu \cdot d \nu$. Therefore, the optical NF is related to the total ASE power $P_{\mathrm{ASE}}$ as follows:

$$
\mathrm{NF}_{\mathrm{EDFA}}=\frac{P_{\mathrm{ASE}}}{h \cdot \nu \cdot \Delta \nu \cdot G} .
$$

\section{PARAMETRIC AmPlifier Under Test}

The FOPA under test is depicted in Fig. 1. The pump is first phase-modulated by a pseudorandom bit sequence of $3.5 \mathrm{~Gb} / \mathrm{s}$ to increase the threshold power of the stimulated Brillouin scattering. Then it is successively amplified by two EDFAs with a filtering of the ASE in between. Finally, a circulator and a reflective fiber Bragg grating (Filter 2) reduce the level of ASE from the EDFAs, as proposed in [1]. A variable attenuator, placed in front of the first EDFA, is used to vary the OSNR of the pump, i.e., the level of the residual ASE at the pump wavelength $\lambda_{p}=$ $1553.3 \mathrm{~nm}$. The pump power remains constant to $640 \mathrm{~mW}$. The amplifying fiber used in this FOPA is a highly nonlinear dispersion-shifted fiber $\left(L=490 \mathrm{~m}\right.$ and $\left.\lambda_{0}=1553 \mathrm{~nm}\right)$. On the signal path, a polarization controller allows adjusting the signal polarization to maximize the gain and an isolator protects the input from occasional reflections. The signal is amplified by an EDFA and its power is controlled with a variable attenuator; it is coupled to the pump through the fiber Bragg grating. At the output of the highly nonlinear fiber, a circulator and a fiber Bragg grating (similar to Filter 2 of the pump) separate the amplification spectral band from the pump. Electrical measurements are performed at the frequency $68 \mathrm{MHz}$, where the pump RIN is below $-155 \mathrm{~dB} / \mathrm{Hz}$. FOPAs are generally pumped by a low power source, amplified by successive EDFAs. First the pump has to be filtered to suppress ASE produced by the EDFAs in the amplification spectral band of the FOPA. But even with the narrowest filtering centered at the pump wavelength, ASE from the EDFAs remains in the bandwidth of the filter at the pump wavelength. When this noise is injected in the parametric amplifier all together with the pump and the signal, it is

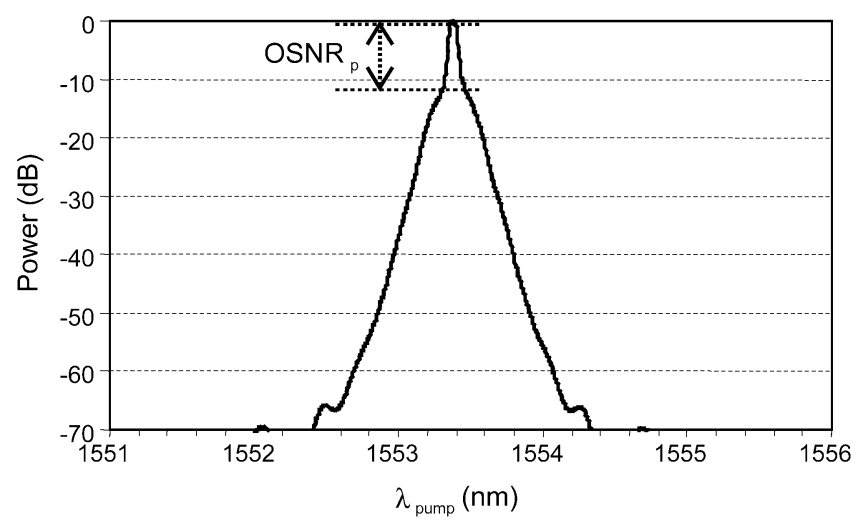

Fig. 2. Input pump spectrum (res $=0.05 \mathrm{~nm}$ ).

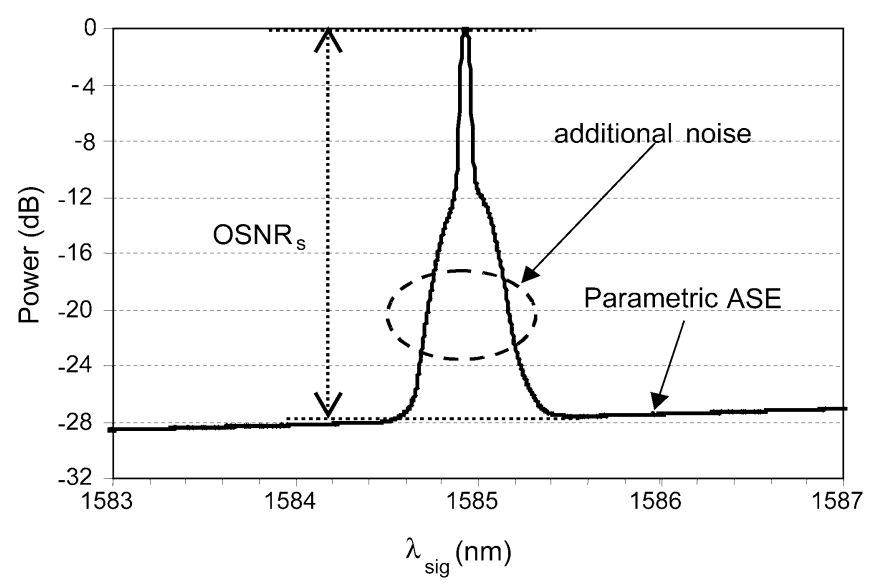

Fig. 3. Example of noise transferred from the pump pedestal (represented in Fig. 2) to the signal wavelength (res $=0.05 \mathrm{~nm}$ ).

reproduced at the signal wavelength through a four-wave mixing (FWM) process between the signal, the pump, and this pump noise. To obtain Fig. 2, we intentionally degrade the FOPA performances by reducing the pump OSNR. In Fig. 3, the transfer of the pump noise to the signal can be observed on the OSA (resolution set to $0.05 \mathrm{~nm}$ ) as an additional noise, very narrow, located around the signal wavelength. Flat broad-band parametric ASE can be seen as well, at a much lower level. With a better pump OSNR, transfer of pump noise at the signal wavelength can be no longer observed experimentally on the OSA so that it is not taken into account in the measurement of OSNRs.

\section{RESULTS AND DISCUSSIONS}

As the noise observed on the OSA is the result of an FWM product between the pump, the signal, and ASE at the pump wavelength, its level is proportional to the signal power and to the level of ASE, i.e., to the inverse of the OSNR of the pump.

These trends have been experimentally verified. The OSNR of the pump and the signal power are controlled with variable attenuators (see Fig. 2). First for a signal wavelength of $1595 \mathrm{~nm}$, the output noise has been measured optically and electrically while the input signal power was increased from -35 to $-5 \mathrm{dBm}$, the pump OSNR remained constant as well as the net gain $(19.1 \mathrm{~dB})$. This measurement was carried out for three different pump OSNRs. Then at the same signal wavelength, the output noise was also measured versus the pump 


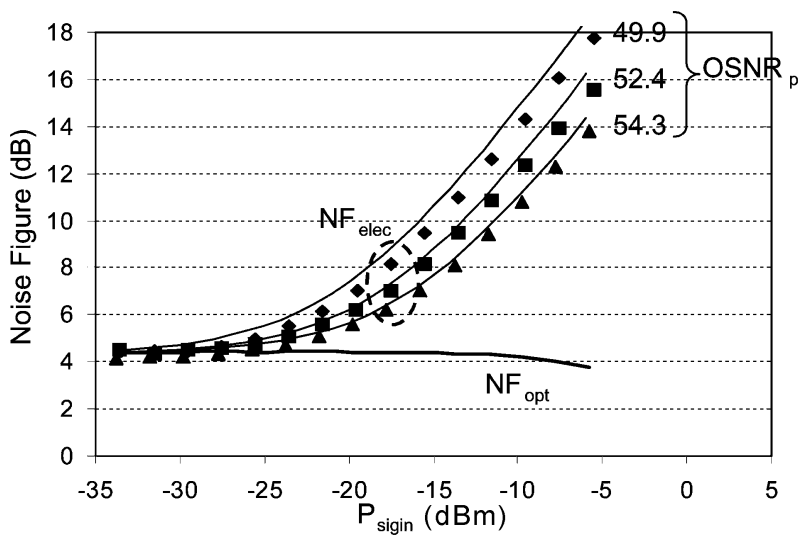

Fig. 4. FOPA NF as a function of signal power for three different pump OSNRs. (Case of $\mathrm{NF}_{\text {elec }}$. Symbols: Eexperiments. Lines: Simulations).

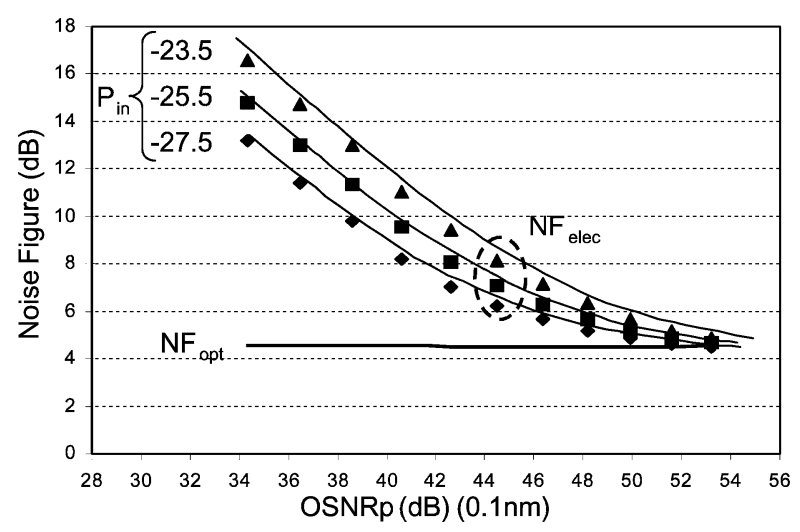

Fig. 5. FOPA NF as a function of pump OSNR for three different signal powers. (Case of $\mathrm{NF}_{\text {elec }}$. Symbols: Experiments. Lines: Simulations).

OSNR in the case of three input signal powers. The resulting NFs are presented in Figs. 4 and 5. In these figures, the electrical and optical NF are represented; for the optical one, the measurements takes into account only parametric ASE. As can be seen, the electrical measurement detects an additional noise, which depends on the input signal power and more particularly on the level of the pump OSNR. This noise gets negligible compared to parametric ASE when the signal power is low and the pump OSNR high. In this case, electrical NF meets optical $\mathrm{NF}$ to $4.4 \mathrm{~dB}$. It is the first time to our knowledge that a real noise floor is electrically measured and that this noise floor corresponds to the optical measurement. This 4.4-dB limit is mainly due to relatively high coupling and distributed losses (respectively, about 0.8 and $0.3 \mathrm{~dB}$ ) and noise produced by the Raman effect [5], [6]. Moreover, we numerically simulated the transfer of pump noise toward the signal through FWM by solving the scalar nonlinear Schrödinger equation with a standard split-step Fourier method. The pump and its noise are modeled by a monochromatic continuous wave, and in the spectral domain, we add a square of random noise centered at the pump wavelength, with a bandwidth of $0.2 \mathrm{~nm}$ and which average level corresponds to the required OSNR. The signal is also a monochromatic continuous wave. Optical waves are all considered in the same state of polarization. The simulated pump OSNR is higher than the experimental one by $3 \mathrm{~dB}$ because pump noise in the orthogonal polarization is not accounted for. At the output of the amplifier, we calculate the level of noise produced around the signal wavelength. The model does not simulate the Raman effect, leading to a minimum NF of $3.8 \mathrm{~dB}$. In experiments, as the pump power remains constant, Raman noise is also constant. A constant noise power was, therefore, added to account for the Raman noise and to achieve the experimental 4.4-dB NF. In Figs. 4 and 5, the simulated results are represented by the lines and the experimental ones by the points. A fair agreement is found between simulations and experimental results, so the numerical model can be used to define a pump OSNR specification. In practical systems, amplifiers provide a total output power of $20 \mathrm{dBm}$, which corresponds, for 128 channels, to a channel input power of $-21 \mathrm{dBm}$. According to our model, a 4.4-dB NF is achieved provided the pump OSNR is higher than $65 \mathrm{~dB}$ $(0.1 \mathrm{~nm})$, in this case, the pump noise is negligible compared to parametric ASE.

\section{CONCLUSION}

An electrical measurement method of the NF of an optical amplifier has been implemented. The results of this measurement were first compared to a standard optical measurement and validated in the case of an EDFA. When applied to an FOPA, it allowed us to experimentally quantify, for the first time, the noise limitations caused by the pedestal noise present at the pump wavelength. The FOPA NF was measured versus the pump OSNR and as a function of the signal power. A numerical model was developed to simulate the transfer of pump noise and validated by these measurements. Using this model, we determine, for practical systems, a minimum required pump OSNR $(0.1 \mathrm{~nm})$ of $65 \mathrm{~dB}$.

\section{REFERENCES}

[1] J. L. Blows and S. E. French, "Low-noise-figure parametric amplifier with a continuous-wave frequency-modulated pump," Opt. Lett., vol. 27, pp. 491-493, 2002.

[2] P. Kylemark, T. Torounidis, P. O. Hedekvist, H. Sunnerud, and P. A. Andrekson, "Noise figure characterization of fiber optical parametric amplifiers," in 29th Eur. Conf. Optical Communication, Rimini, Italy, 2003, Paper We.1.6.4.

[3] P. Kylemark, P. O. Hedekvist, H. Sunnerud, M. Karlsson, and P. A. Andrekson, "Noise characteristics of fiber optical parametric amplifiers," $J$. Lightw. Technol., vol. 22, no. 2, pp. 409-416, Feb. 2004.

[4] F. W. Willems, J. C. van der Plaats, C. Hentschel, and E. Leckel, "Optical amplifier noise figure determination by signal RIN subtraction," in Tech. Dig. Symp. Optical Fiber Measurements, Sep. 1994, pp. 7-9.

[5] P. L. Voss and P. Kumar, "Raman-noise-induced noise-figure limit for $\chi(3)$ parametric amplifiers," Opt. Lett., vol. 29, no. 5, pp. 445-447, 2004.

[6] R. Tang, P. L. Voss, J. Lasri, P. Devgan, and P. Kumar, "Noise-figure limit of fiber-optical parametric amplifiers and wavelength converters: Experimental investigation," Opt. Lett., vol. 29, pp. 2372-2374, 2004. 